\title{
An Overview of Metamaterials Used in Applicators in Hyperthermia Cancer Treatment Procedure
}

\author{
Nabilah Abdul Jaffar, Kasumawati Bt Lias*, Nina Korlina Madzhi, Norlida Buniyamin \\ Faculty of Electrical Engineering, Universiti Teknologi MARA (UiTM), 40450 Shah Alam, Selangor, Malaysia \\ nabilah_ajmb@yahoo.com,nbuniyamin@salam.uitm.edu.my \\ *Faculty of Engineering, Universiti Malaysia Sarawak(Anima's), 94300, Kota Samarian, Sarawak, Malaysia
}

\begin{abstract}
This paper presents an overview of the usage of metamaterial slabs and lens in applicators for hyperthermia cancer treatment procedure. The evolution of metamaterials starts from 2007 until 2017 was traced. The review indicates that Left handed metamaterial (LHM) lens when integrated with an antenna can improve focusing capabilities of the antenna used as an applicator to treat the cancerous area. However, current applicators have poor focal spot when directed towards the actual tumour area. Subsequently, a proposed modified applicator that integrates Left-Handed Metamaterial (LHM) lens with existing antenna is discussed. The proposed antenna termed LHMA is expected to have an improved focusing capabilities which could be used to kill cancerous tissues without severely affecting surrounding healthy tissues. The goal of the research is to reduce unwanted hot-spots developed using current available applicators. The focusing abilites of the proposed appicator will be evaluated using the Specific Absorption Rate (SAR).
\end{abstract}

Keywords- Left-handed Metamaterial (LHM); Non-invasive, Hyperthermia, Specific Absorption Rate (SAR)

\section{INTRODUCTION}

Interest in the development of antennas or sometimes termed as applicator for non-invasive hyperthermia cancer treatment (NIHCT) procedure has recently grown tremendously. This is because NICHT could destroy cancerous cells without severely affecting patients as opposed to other cancer treatment.

Nowadays, breast cancer contributes to the highest number on cancer incidences among women all over the year. In Malaysia specifically, according to [1], women are the most at risk of cancer involving 10,290 patients out of 100,000 people in the year 2016 where it is the highest incidence with $14.5 \%$, if compared to other cancer incidences such as lung, stomach and leukemia.

Cancer is a group of diseases involving uncontrolled division of abnormal cell growth that forms malignant tumor is that can then invade nearby parts of the body or spread to other organs. Cancerous cells can be killed by various cancer treatment methods such as radiotherapy, chemotherapy, hormone therapy and surgery [2]. However these methods are invasive and will harm the patient. For example surgery will cause physical pain and chemotherapy will cause pain due to chemicals injected in the bodies.

An alternative treatment is by the use of microwave hyperthermia which has to a certain extent been considered as an effective treatment for cancer therapy. Hyperthermia is also called thermal therapy or thermotherapy is a type of cancer treatment in which body tissue is exposed to high temperature. It is a treatment which requires high temperature around $41^{\circ} \mathrm{C}-45^{\circ} \mathrm{C}$ in order to provide a denaturation into a cancerous tissue which then will be induced to cell death with minimal side effects [3],[4]. Hyperthermia can work alone or commonly as an adjuvant towards chemotherapy and radiotherapy.

Hyperthermia can be presented either invasive or noninvasive. According to [5], non-invasive hyperthermia cancer treatment is safer and effective treatment to fight against various types of cancer with less pain and injury. Therefore, many researchers focused on non-invasive hyperthermia antenna/applicator recently. The applicator is considered as the main part of hyperthermia that it used to deliver electromagnetic energy which will transform as heat and then distributed and absorbed by the cancerous tissues at a certain level of heat and time.

The use of an appropriate antenna is very crucial as heat generated by the antenna Electromagnetic (EM) fields will be used to kill/destroy the cancerous tissues or cells. Various researches were conducted to obtain the quality of hyperthermia treatment from the perspective antenna design. Currently, research in the establishment of an antenna with the integration of metamaterial slab and lens has grown rapidly as depicted in Figure 1.

\section{METAMATERIAL}

Metamaterial technology was further developed to lenses, where LHM slab can act as a lens that will focus the incident waves. Due to its unique characteristics, Pendry who 